loss of crystals occurring either during tissue preparation or in vivo. Crystals were situated either randomly in the matrix, many of them within a few micrometers of the articular surface, or around the cells. Although the number of crystals varied, this did not seem to be related to the time interval between the fracture and the sampling of the tissue.

$X$-ray emission microanalyses of crystals $(\mathrm{n}=10)$ in formalin fixed sections showed calcium and phosphorus. The ratio of calcium to phosphorus, uncorrected for absorption, fluorescence, and atomic weight was 1.95 (SD 0.30), suggestive of apatite rather than pyrophosphate deposits.
Ultrastructurally articular surfaces were often roughened, though smooth to the naked eye, and showed infiltration with electron dense material. They were only rarely fissured. In many specimens the superficial collagen fibrils retained the normal density of packing and orientation tangential to the surface. More deeply, the fibres could show some disorganisation. Flattened chrondrocytes were often present in the superficial zone. Chrondrocytes in deeper tissue contained irregular lobed nuclei and several lipid droplets in addition to active Golgi and endoplasmic reticulum.

Crystal deposition in the hip joints of these women may be related to osteoporosis, and this requires further investigation. Localisation of the crystals within the cartilage (superficial zone and pericellular regions) corresponds to sites of extracellular lipid in aging articular cartilage generally. Perhaps the most noteworthy features are the occurrence of crystals in non-arthritic hip joints, and their occurrence in all the elderly women in this group.

\section{References}

1 Dieppe $\mathrm{P}$ A. New knowledge of chrondrocalcinosis. J Clin Path 1978; 31, suppl 12: 214-22.

2 Ali S Y. In: Ascenzi E, Bonucci E, de Bernard B, eds. Matrix vesicles. Milan: Wichtig, 1981: 241-7.

\title{
Ultrastructural studies of pyrophosphate crystal deposition in articular cartilage
}

\author{
S. Y. ALI ${ }^{1} S$. GRIFFITHS,${ }^{1} M$. T. BAYLISS,${ }^{1}$ AND P. A. DIEPPE ${ }^{2}$ \\ From the ${ }^{1}$ Institute of Orthopaedics, Royal National Orthopaedic Hospital, Stanmore, Middlesex, and ${ }^{2}$ Bristol Royal \\ Infirmary, Bristol
}

The mechanism of calcium pyrophosphate crystal formation in the articular cartilage of patients suffering from this crystal deposition disease has not yet been fully elucidated. It has been suggested that the earliest pyrophosphate crystal deposition is adjacent to chondrocyte lacunae ${ }^{1}$ and has been supported by light microscopical studies. Ultrastructural studies have also been performed on cartilage from patients with chondrocalcinosis but they have been restricted to the study of the changes in the matrix. ${ }^{23}$ We have embarked on an electron microscopical study of articular cartilage specimens from patients with calcium pyrophosphate crystal deposition disease and have combined it with electron probe analysis of the crystals to determine their chemical composition.

By using modified staining techniques it is now possible to show crystals in ultrathin sections of cartilage. Large islands of crystals were seen in the matrix, confirming earlier

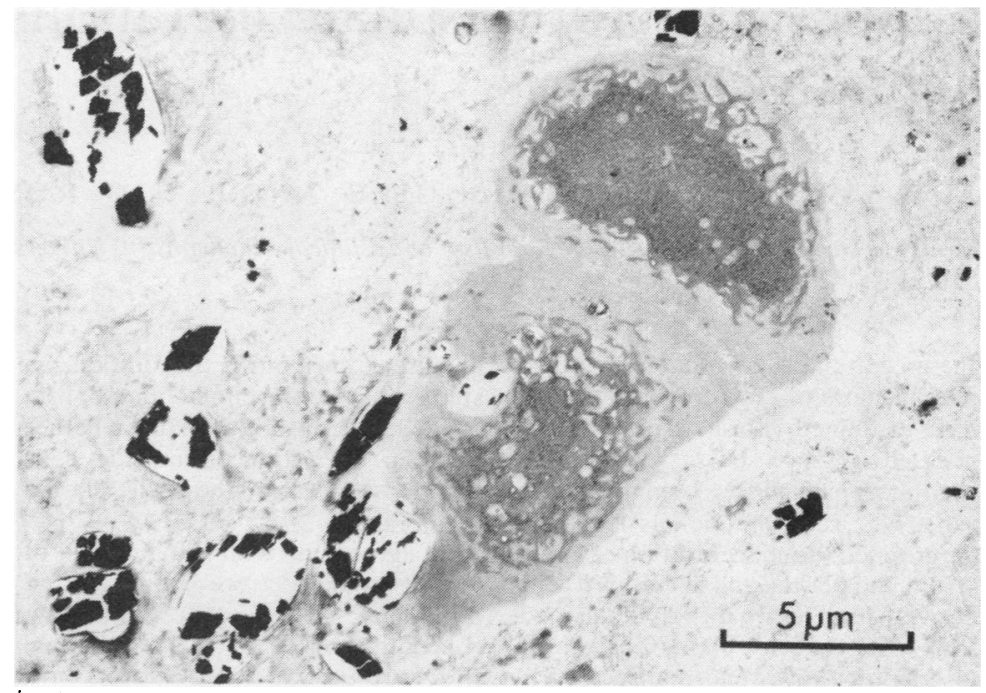

Fig. 1 Electron microscopical picture of two chondrocytes in the matrix of articular cartilage specimen obtained from the knee of a patient with calcium pyrophosphate crystal deposition disease ( 70 year old woman). Note that some of the crystals are adjacent to the chondrocyte and are present within the lacuna. Both the cells have an abnormal amount of glycogen in the cytoplasm. The appearance of the matrix is also abnormal. $\times \overline{5} \overline{5} \tilde{0} \dot{0}$ (original magnification). 
observations on light microscopy. More interestingly, smaller crystals were seen pericellularly and in the intercellular matrix. Some crystals were seen adjacent to the chondrocytes and located in the lacuna (Fig. 1). Electron probe analysis of the crystals in various locations, and differing in size, gave a consistent calcium to phosphorus ratio of approximately $1 \cdot 15: 1$ similar to that of the calcium pyrophosphate standard. This ratio is quite different and much lower than the ratio of calcium and phosphorus in the three different types of apatite crystals found in human osteoarthritic articular cartilage. ${ }^{45}$

There were various non-specific changes in the cells and in the matrix components that were indicative of a rapidly degenerating cartilaginous tissue. Thus there were focal changes in the staining of the collagen and proteoglycan particles which gave the matrix, in some areas, a more granular appearance unlike the ordered, fibrous nature of normal cartilage matrix. In other areas there was a band-like, collagenous encapsulation of the lacuna area, which contained several cells in a cluster. Extracellular matrix vesicles were absent in many areas but weré sometimes seen pericellularly in focal areas where chondrocyte degeneration and necrosis was evident.

In terms of chondrocyte morphology two changes were particularly noticeable. Firstly, some cells showed abnormally large islands of glycogen in the cytoplasm (Fig. 1). Secondly, other chondrocytes showed abnormal increase in the amount of rough endoplasmic reticulum. If these observations are true it may imply increased synthesis of glycogen and other cartilage constituents. Inorganic pyrophosphate is a byproduct of many metabolic activities, and biosynthetic pathways, and this has been implicated by others as a mechanism for the formation of excess pyrophosphate in some tissues and fluids. From our observations we can only suggest that the calcium pyrophosphate crystals seen in the vicinity of chondrocytes may be formed by the interaction of a cellular product and some matrix ion or component. Further studies on the quantitative analysis of the level of pyrophosphatase-type enzymes in normal and degenerative cartilage are under way to see if the accumulation of pyrophosphate in the tissue is due to the lack of a specific degradative enzyme.

\section{References}

1 McCarty D J. Pseudogout. In: Hollander J L, McCarty D J, eds. Arthritis and allied conditions. Philadelphia: Lea and Febiger, 1972: 1140-60.

2 Bjelle A O, Sundstrom B K G. An ultrastructural study of the articular cartilage in calcium pyrophosphate dihydrate (CPPD) crystal deposition disease (chondrocalcinosis articularis). Calif Tiss Res 1975; 19: 63-71.

3 Schumacher H R. Ultrastructural findings in chondrocalcinosis and pseudogout. Arthritis Rheum 1976; 19: 413-25.

4 Ali S Y, Griffiths S. New types of calcium phosphate crystals in arthritic cartilage. Semin Arthritis Rheum 1981; 11, suppl 1: 124-6.

5 Ali S Y. New knowledge of osteoarthritis. $J$ Clin Pathol 1978; 31, suppl 12: 191-9.

\title{
Studies of pyrophosphate metabolism in relation to chondrocalcinosis
}

\author{
A. M. CASWELL, M. K. B. McGUIRE, AND R. G. G. RUSSELL \\ From the Department of Human Metabolism and Clinical Biochemistry, University of Sheffield Medical School, Sheffield \\ S10 2RX
}

In an attempt to understand the pathogenesis of chondrocalcinosis we have examined various aspects of the metabolism of inorganic pyrophosphate $\left(\mathrm{PP}_{\mathrm{i}}\right)$. Using a highly sensitive radiometric assay, ${ }^{1}$ we determined the concentration of $\mathrm{PP}_{\mathrm{i}}$ in serum and plasma and in cultured cells derived from normal individuals and from patients with chondrocalcinosis to identify any abnormality in $\mathrm{PP}_{\mathrm{i}}$ metabolism. We also examined the intracellular metabolism of $\mathrm{PP}_{\mathrm{i}}$ in cultured articular chondrocytes and meniscus cells derived from normal individuals to define the factors that influence the intracellular production and breakdown of $\mathrm{PP}_{\mathrm{i}}$. In addition, we studied the extracellular metabolism of $\mathrm{PP}_{\mathrm{i}}$ by these cells to define whether $\mathrm{PP}_{\mathrm{i}}$ can be produced outside cells and/or can cross the cell plasma membranes.

In agreement with the findings of earlier studies, ${ }^{23}$ we observed no differences in the serum and plasma concentrations of $\mathrm{PP}_{1}$ between normal individuals and patients with chondrocalcinosis. Similarly, in cultured skin fibroblasts, we failed to observe any difference in the content of $\mathrm{PP}_{\mathrm{i}}$ between cells derived from normal individuals and those derived from patients with chondrocalcinosis.
In contrast, the amounts of $\mathrm{PP}_{\mathrm{i}}$ in cultured articular chondrocytes and meniscus cells derived from patients with chondrocalcinosis were substantially higher than the $P P_{i}$ contents of these cell types derived from normal individuals, especially during primary culture. However, this may not necessarily reflect a change in intracellular $\mathrm{PP}_{\mathrm{i}}$ metabolism in these cell types in this condition, as it may be due to the continued presence of calcium pyrophosphate crystals that were present in vivo. For example, in one such culture derived from a patient with chondrocalcinosis crystals resembling triclinic calcium 